\title{
Non-Target Non-Enhancing Lesion Response
}

National Cancer Institute

\section{Source}

National Cancer Institute. Non-Target Non-Enhancing Lesion Response. NCI Thesaurus.

Code C135481.

An assessment of the response to the therapy of the non-target non-enhancing lesion. 